\title{
NONLINEAR VIBRATIONS OF ORTHOGONAL MECHANISM OF SHAKING TABLE
}

\author{
K. BISSEMBAYEV and Z. ISKAKOV* \\ Laboratory of Vibratory Mechanisms and Machinery \\ U.A. Dzholdasbekov Institute of Mechanics and Machine Science \\ Kurmangazy street, 29, Almaty, KAZAKHSTAN \\ E-mail: iskakov53@mail.ru
}

\begin{abstract}
The dynamics of orthogonal mechanism in the presence of a shaking table of fixed cargo is studied. The focus is on the vibrational motion of the mechanism in slow-speed motor rotation. The analysis of the solutions for motion nonlinear equations showed that the angular velocity of the guiding link oscillates according to the harmonic law about a mean value. The character of changing its amplitude, depending on the weight of the cargo and the length of the guiding link, is determined. Dependence of the mean angular velocity on the motor torque is set.
\end{abstract}

Key words: orthogonal mechanism, dynamics, asymptotic method, essentially nonlinearity, stability.

\section{Introduction}

In recent years, the vibrating equipment in the mechanical engineering industry has begun to be constructed on the basis of leverage mechanisms. These mechanisms possess unique abilities to create oscillations of the executive element. The development of vibration mechanisms that are based on mathematical modelling results in acceptable and practical results.

The structural schemes of vibration machines, as a rule, are not complex; however, you need to determine their parameters accurately for a successful application. These parameters can only be determined based on researching the dynamics of the vibrating machines and the technological processes performed by these machines.

In the work of Azbel et al. (1981) presents a study of vibration transportation in a material part, and in summary, this research presents the vibration transportation through solid substances and the behavior of granular materials and continuous medium undergoing vibration.

Baksys and Liutkauskiene (2010) examine the horizontal motion of a part in two directions perpendicular to the excited plane while controlling the dry friction coefficient between the plane and the part. The dependences of the transition of the part from its initial position to the center of the stable motion path were defined. Additionally, the dependences of the directional angle of the motion path from the moment when friction was reduced relative to the excitation signal and the duration of time of the decreased friction were defined. Baksys and Liutkauskiene (2008) cite research that features the vibration transition of a body on a swinging plane, which is applicable to the field of automated part collection. Dynamic and mathematical models of vibration transition were constructed. The operating conditions of the body were defined as being dependent on the excitation frequency and the oscillation of the plane, the rolling angle of the plane and the factor of rigidity.

Dorinin and Danshin (1993) and Feng et al. (2004) examine the tasks of the optimal and dynamic synthesis of a swivel-lever guidance mechanism and counterbalancing, and the solution to these tasks helps determine the directional impact of the lever vibrator on a foundation. Numerical interpretation is

\footnotetext{
* To whom correspondence should be addressed
} 
used to determine the combined target function that contains all conditions that achieve complete dynamic balancing.

Balancing of the principal moment of inertia forces based on the mean-square approximation was used in Arakelian and Daham (2001), where the counterweights were located on links associated with a rack.

Hong-Sen Yan and Ren-Chung Soong (2001) present a new method of determining the four links, which, in turn, satisfies all kinematic demands and ensure dynamic balance. The predicted characteristics of the driving (working) link motion and the conditions of dynamic balance are ensured by means of (prescribing) the driven link speed change, the disk counterweights and the sizes of the links.

The effectiveness of all the above-mentioned methods is primarily determined by the choice of the model and the oscillating conditions for the working body of the corresponding machine, because the most diverse and complicated types of oscillations, such as harmonic, polyharmonic, rectilinear, two-component and spatial oscillations are used in practice. These modes may be implemented with the help of leverage mechanisms which have a wide range of functional abilities.

One of the vibrating pieces of equipment, a shaking table with flat leverage mechanisms, can be successfully utilized in the construction industry to compact concrete mixtures, in the chemical, pharmaceutical and food industries to apply vibrating impact on pulps and suspensions, in the mining industry for screening fractions depending on the volume and weight, and for many other purposes. One of the problems with the mathematical modelling during the development of vibrating equipment based on leverage mechanisms is the variability of the characteristics of such mechanisms. An apparatus of generalized functions is used in Drakunov and Tuleshov (2007) and Tuleshov and Tuleshov (2012) to determine the solution of equations describing the machine assembly dynamics.

Bissembayev and Iskakov (2012) studied an oscillation of the automatic press shaking table that uses flat leverage mechanisms. A mathematical model of the automatic press shaking table that uses an orthogonal mechanism was also developed.

Kononenko (1964) reviews linear and non-linear systems, and it is hypothesized that they are influenced and excited by sources of energy that have a limited capacity.

Further development and presentation of the characteristics of oscillating systems that interact with the energy source were obtained in the work in Alifov and Frolov (1985). This work cites the dynamic analysis of a self-oscillating system interacting with the energy sources in the presence of non-linear elastic constraints, periodic, parameter impacts and delays. Non-linear forced and parameter oscillations of the systems interacting with two energy sources are examined.

Krivtsov (2000) examines stationary motions of a non-symmetrical spin (unbalanced rotor), which has a fixed point and is impacted by the moment of elasticity and the torque. The impact of the dissipation created by the engine with restricted power on the stability of the conservative system is studied.

The purpose of this work is to study the dynamics of the orthogonal mechanism of a shaking table with a low-speed engine source in the presence of a fixed load (account of load) on the operating link.

\section{Kinematic relations}

The calculation model for the orthogonal mechanism is shown in Fig.1. The start of the $O X Y$ coordinate system is placed at the crank rotation axis. Here we designate the coordinates of the articulated link C (Fig.1) through $X$ and $Y$, and the ranges of the horizontal and the vertical oscillations of the orthogonal mechanism are designated as $a_{1}$ and $a_{2}$, respectively. The following kinematic relations (Tuleshov and Tuleshov, 2012) can be recorded from the equations of the closeness of the vector contours as projections onto the coordinate axes. 


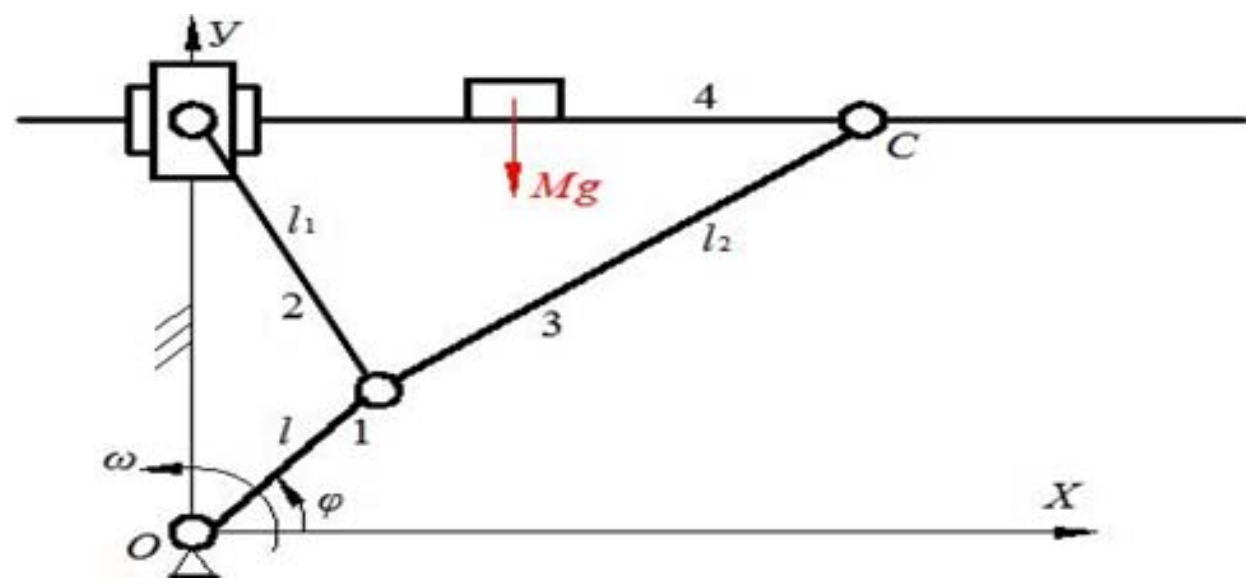

Fig.1. A schematic diagram of the orthogonal mechanism of the shaking table.

$$
\begin{aligned}
& X=l \cos \varphi+l_{2} \sqrt{1-\frac{l_{1}^{2}}{l_{2}^{2}}+\frac{l^{2}}{l_{2}^{2}} \cos ^{2} \varphi} \\
& Y=l \sin \varphi+l_{1} \sqrt{1-\frac{l^{2}}{l_{1}^{2}} \cos ^{2} \varphi}
\end{aligned}
$$

where $l, l_{1}, l_{2}$ and $l_{3}$ are the lengths of links $1,2,3$ and 4 respectively and $\phi$ is the - angle of the crank rotation axis (Fig.1).

The extreme values of the coordinates $x$ and $y$ are

$$
\begin{aligned}
& X_{\max }=X(0)=l+l_{2} \sqrt{1-\frac{l_{1}^{2}}{l_{2}^{2}}+\frac{l^{2}}{l_{2}^{2}}}, \\
& X_{\min }=X(\pi)=-l+l_{2} \sqrt{1-\frac{l_{1}^{2}}{l_{2}^{2}}+\frac{l^{2}}{l_{2}^{2}}}, \\
& Y_{\max }=l+l_{1}, \\
& Y_{\min }=-l+l_{1} .
\end{aligned}
$$

Average values of the coordinates $X$ and $Y$ are

$$
\begin{aligned}
& X_{m e}=\frac{X_{\max }+X_{\min }}{2}=l_{2} \sqrt{1-\frac{l_{1}^{2}}{l_{2}^{2}}+\frac{l^{2}}{l_{2}^{2}}}, \\
& Y_{m e}=\frac{Y_{\max }+Y_{\min }}{2}=l_{l} .
\end{aligned}
$$


The ranges of the horizontal and the vertical oscillations of the articulated link $C$ of the orthogonal mechanism relative to the average values of the coordinates are determined by the following formulas

$$
\begin{aligned}
& a_{1}=X_{\max }-X_{m e}=X_{m e}-X_{\min }=l, \\
& a_{2}=Y_{\max }-Y_{m e}=Y_{m e}-Y_{\min }=l .
\end{aligned}
$$

From these formulas, it is shown that the ranges of the horizontal and the vertical oscillations coincide.

We transfer the start of the $O X Y$ coordinate system to the point $\left(X_{m e}, Y_{m e}\right)$, i.e., transform the $O X Y$ coordinate system into the $O^{\prime} \xi \eta$ coordinate system

$$
\begin{aligned}
& X=X_{m e}+\xi, \\
& Y=Y_{m e}+\eta
\end{aligned}
$$

from which

$$
\begin{aligned}
& \xi=l \cos \varphi-l_{2}\left[\sqrt{1-\frac{l_{1}^{2}}{l_{2}^{2}}+\frac{l^{2}}{l_{2}^{2}}}-\sqrt{1-\frac{l_{1}^{2}}{l_{2}^{2}}+\frac{l^{2}}{l_{2}^{2}} \cos ^{2} \varphi}\right], \\
& \eta=l \sin \varphi-l_{l}\left[\sqrt{1-\frac{l^{2}}{l_{2}^{2}}}-\sqrt{1-\frac{l^{2}}{l_{1}^{2}} \cos ^{2} \varphi}\right] .
\end{aligned}
$$

\section{Dynamics of mechanism movement}

\subsection{The equation of the mechanism motion}

The equation of the orthogonal mechanism motion with the presence of a fixed counterweight on the horizontal link (link 4) is obtained in Bisembayev and Iskakov (2012). It has the following form

$$
\begin{aligned}
& A_{0} \ddot{\varphi}+\left[A_{3}\left(\frac{1}{2} \cos ^{3} \varphi-\sin ^{2} \varphi \cos \varphi-\sin \varphi \cos \varphi\right)+f M l^{2} \sin \varphi\right] \dot{\varphi}^{2}+P \cos \varphi+ \\
& +k M_{D} \cos \varphi \sin \varphi=M_{D}-f M g l-k M_{D}
\end{aligned}
$$

where

$$
\begin{aligned}
& A_{0}=M\left(1+\frac{m_{3}}{M}\right) l^{2}+J ; \quad A_{3}=2 M l^{2} \frac{l}{l_{1}} \\
& P=M\left[1+\frac{m_{1}}{M}+\frac{m_{2}}{M}+\frac{m}{2 M}\right] g l, \quad J=\frac{1}{3} m l^{2}
\end{aligned}
$$


where $M$ - counterweight mass , $m, m_{1}, m_{2}$ and $m_{3}$ - mass of the links $1,2,3$ and $4 ; J$ - link moment of inertia $1 ; f$ - coefficient of sliding friction; $k$ - coefficient of friction, rotation; $M_{D}$ - moment of the driving forces.

We consider the case where there is no friction, i.e., the case when $f=0$ and $k=0$. By substituting $d \tau=\omega_{0} d t$ and using some trigonometric relations, we transform the equation of motion (3.1) to immeasurable form

$$
\begin{aligned}
& \frac{d^{2} \varphi}{d \tau^{2}}+\varepsilon(\cos \varphi-4 \sin 2 \varphi+3 \cos 3 \varphi)\left(\frac{d \varphi}{d \tau}\right)^{2}+\cos \varphi=\frac{M_{D}}{\omega_{0}^{2} A_{0}}, \\
& \omega_{0}{ }^{2}=\frac{P}{A_{0}} ; \quad \varepsilon=\frac{A_{3}}{A_{0}} .
\end{aligned}
$$

The impact of non-ideal energy source on the oscillating system has to be expressed as a function $M(\varphi, \dot{\varphi})$, where $\varphi$ - coordinate of movement of the energy source. The torque on the shaft of a motor (e.g., a DC motor with shunt excitation) is determined according to the formula

$$
M_{D}=a-b \dot{\varphi}
$$

where $a$ and $b$-constant coefficients depending on the parameters of the engine.

Now we assume that the limited power engine is slow-motion, i.e., the velocity of rotational motion of the motor shaft is equal to several units, thus we impose conditions $b \gg \omega_{0} A_{0}$. Let us substitute (3.3) and (3.2) and obtain the motion equation as

$$
\frac{d^{2} \varphi}{d \tau^{2}}+\frac{b}{\omega_{0} A_{0}} \frac{d \varphi}{d \tau}+\varepsilon(\cos \varphi-4 \sin 2 \varphi+3 \cos 3 \varphi)\left(\frac{d \varphi}{d \tau}\right)^{2}+\cos \varphi=\frac{a}{\omega_{0}^{2} A_{0}}
$$

Equation (3.4) is essentially nonlinear, as a nonlinear term is included to the equation without a small parameter.

\subsection{Solution of the motion equation by the asymptotic method}

We now turn to the solution of the motion Eq.(3.2) by the asymptotic method (Gulyayev et al., 1989). The static equilibrium of the mechanism is defined by the equation

$$
\cos \varphi=\frac{a}{\omega_{0}^{2} A_{0}}
$$

Hence, we find the condition of rotational motion excitation in the form

$$
\frac{a}{\omega_{0}^{2} A_{0}} \succ 1
$$

Substituting 


$$
\varphi=z, \quad \dot{z}=\Omega+x
$$

We bring Eq.(3.4) to the form

$$
\begin{aligned}
& \dot{x}=-\frac{b}{\omega_{0} A_{0}} x-\varepsilon(\cos z-4 \sin 2 z+3 \cos 3 z)(\Omega+x)^{2}-\cos z, \\
& \dot{z}=\Omega+x,
\end{aligned}
$$

where

$$
\Omega=\frac{a}{\omega_{0} b}
$$

Suppose that $\tau=\mu^{2} s$, where $\mu=\frac{\omega_{0} A_{0}}{b}$. Then the equitation system (3.8) results in

$$
\begin{aligned}
& x^{\prime}=-\mu x-\mu^{2} \varepsilon(\cos z-4 \sin 2 z+3 \cos 3 z)(\Omega+x)^{2}-\mu^{2} \cos z, \\
& z^{\prime}=\mu^{2} \Omega+\mu^{2} x,
\end{aligned}
$$

where we have notation $x^{\prime}=\frac{d x}{d s}$.

For the system (3.9) we agree to consider the following Cauchy problem

at

$$
s=0, \quad z=0, \quad z^{\prime}=0, \quad x=\bar{x} .
$$

Following Bogolyubov and Mitropolskiy (1974), Moyseyev (1969), we suppose

$$
\begin{aligned}
& x=\bar{x}+\mu u_{1}(\bar{x}, \bar{z})+\mu^{2} u_{2}(\bar{x}, \bar{z})+\mu^{3} u_{3}(\bar{x}, \bar{z})+\ldots \\
& z=\bar{z}+\mu v_{l}(\bar{x}, \bar{z})+\mu^{2} v_{2}(\bar{x}, \bar{z})+\mu^{3} v_{3}(\bar{x}, \bar{z})+\ldots
\end{aligned}
$$

where functions $\bar{x}$ and $\bar{z}$ satisfy the following equations

$$
\begin{aligned}
& \bar{x}^{\prime}=\mu A_{1}(\bar{x})+\mu^{2} A_{2}(\bar{x})+\mu^{3} A_{3}(\bar{x})+\ldots \\
& \bar{z}^{\prime}=\mu^{2} \Omega+\mu B_{l}(\bar{x})+\mu^{2} B_{2}(\bar{x})+\mu^{3} B_{3}(\bar{x})+\ldots
\end{aligned}
$$

We assume that the functions $u_{i}, v_{i}, \frac{\partial u_{i}}{\partial \bar{x}}$ and $\frac{\partial v_{i}}{\partial \bar{x}}$ are limited. Further, in order to satisfy the conditions (3.10), we take 


$$
u_{i}(\bar{x}, 0)=v_{i}(\bar{x}, 0)=0 ; \quad \frac{\partial u_{i}(\bar{x}, 0)}{\partial \bar{x}}=\frac{\partial v_{i}(\bar{x}, 0)}{\partial \bar{x}}=0
$$

Taking into account Eq.(3.12), we expand the functions $\cos z, \sin 2 z$ and $\cos 3 z$ on small parameters $\mu$ to the Taylor series

$$
\begin{aligned}
& \cos z=\cos \bar{z}-\mu v_{1} \sin \bar{z}-\frac{1}{2} \mu^{2}\left(v_{1}^{2} \cos \bar{z}+2 v_{2} \sin \bar{z}\right)+ \\
& +\frac{1}{6} \mu^{3}\left(v_{1}^{3} \sin \bar{z}-6 v_{1} v_{2} \cos \bar{z}-6 v_{3} \sin \bar{z}\right)+\ldots, \\
& \sin 2 z=\sin 2 \bar{z}+2 \mu v_{1} \cos 2 \bar{z}+2 \mu^{2}\left(-v_{1}^{2} \cos 2 \bar{z}+v_{2} \cos 2 \bar{z}\right)- \\
& -\frac{2}{3} \mu^{3}\left(2 v_{1}^{3} \cos 2 \bar{z}+6 v_{1} v_{2} \sin 2 \bar{z}-3 v_{3} \cos 2 \bar{z}\right)+\ldots, \\
& \cos 3 z=\cos 3 \bar{z}-3 \mu v_{1} \sin 3 \bar{z}+\frac{3}{2} \mu^{2}\left(-3 v_{1}^{2} \cos 3 \bar{z}+2 v_{2} \sin 3 \bar{z}\right)+ \\
& +\frac{3}{2} \mu^{3}\left(3 v_{1}^{3} \sin 3 \bar{z}-2 v_{1} v_{2} \cos 3 \bar{z}+2 v_{3} \sin 3 \bar{z}\right) \text {. }
\end{aligned}
$$

Equations for determining the function $u_{i}, \mathrm{v}_{i}, \mathrm{~A}_{i}$ and $B_{i}$ are as follows

1.

$$
A_{1}=-\bar{x}
$$

2.

$$
B_{1}=0
$$

3.

$$
\bar{x} \frac{\partial u_{1}}{\partial \bar{x}}-u_{1}=A_{2}+\cos \bar{z}+\varepsilon(\Omega+\bar{x})^{2}(\cos \bar{z}-4 \varepsilon \sin 2 z+3 \cos 3 \bar{z})
$$

4. $\quad \bar{x} \frac{\partial v_{1}}{\partial \bar{x}}=B_{2}-\bar{x}$

5.

$$
\begin{aligned}
& \bar{x} \frac{\partial u_{2}}{\partial \bar{x}}-u_{2}=A_{3}+\frac{\partial u_{1}}{\partial \bar{x}} A_{2}+\left(\Omega+B_{2}\right) \frac{\partial u_{1}}{\partial \bar{z}}-\varepsilon \mathrm{v}_{1}(\Omega+\bar{x})^{2}(\sin \bar{z}+8 \cos 2 \bar{z}+9 \sin 3 \bar{z})+ \\
& +2 \varepsilon u_{1}(\Omega+\bar{x})(\cos \bar{z}-4 \sin 2 \bar{z}+3 \cos 3 \bar{z})-v_{1} \sin \bar{z} ;
\end{aligned}
$$

6.

$$
\bar{x} \frac{\partial v_{2}}{\partial \bar{x}}=B_{3}+\frac{\partial v_{1}}{\partial \bar{x}} A_{2}+\left(\Omega+B_{2}\right) \frac{\partial v_{1}}{\partial \bar{z}}-u_{1}
$$

Let us consider the third Eq.(3.14). From condition (3.13) we find

$$
A_{2}=-\left[1+4 \varepsilon(\Omega+\bar{x})^{2}\right] .
$$


Substituting $u_{1}=\bar{x} \cdot \psi$ we bring Eq.(3.14) $)_{3}$ to the form

$$
\begin{aligned}
& \frac{\partial \psi}{\partial \bar{x}}=-\frac{1}{\bar{x}^{2}}-4 \varepsilon\left(\frac{\Omega^{2}}{\bar{x}^{2}}+\frac{2 \Omega}{\bar{x}}+1\right)+ \\
& +\varepsilon(\cos \bar{z}-4 \sin 2 \bar{z}+3 \cos 3 \bar{z}) \cdot\left(\frac{\Omega^{2}}{\bar{x}^{2}}+\frac{2 \Omega}{\bar{x}}+1\right)+\frac{1}{\bar{x}^{2}} \cos \bar{z}
\end{aligned}
$$

Consequently, the solution of Eq.(3.14) 3 takes the form

$$
u_{1}(\bar{x}, \overline{\mathrm{z}})=1-\cos \bar{z}+\varepsilon\left(\Omega^{2}-2 \Omega \bar{x} \ln \bar{x}-\bar{x}^{2}\right)(4-\cos z+4 \sin 2 \bar{z}-3 \cos 3 \overline{\mathrm{z}})+C_{1}(\overline{\mathrm{z}}) \cdot \bar{x}
$$

where $C_{l}(\bar{z})$ - arbitrary function $\bar{z}$. However, the condition (3.13) immediately gives $C_{l}(\bar{z})=0$. Repeating the argument for the fourth equation of (3.14), we obtain

$$
B_{2}=\bar{x}
$$

and hence

$$
\mathrm{v}_{1}=0
$$

Using the results (3.16), (3.17) and (3.18), rewrite the fifth equation of (3.14) in the form

$$
\bar{x} \frac{\partial u_{2}}{\partial \bar{x}}-u_{2}=A_{3}-\left[1+4 \varepsilon(\Omega+\bar{x})^{2}\right] \frac{\partial u_{1}}{\partial \bar{x}}+(\Omega+\bar{x}) \frac{\partial u_{1}}{\partial \bar{z}}
$$

whence

$$
A_{3}=-8 \varepsilon(\Omega+\bar{x})\left(\Omega^{2}+2 \Omega \bar{x} \ln \bar{x}-\bar{x}^{2}\right)
$$

and therefore

$$
\begin{aligned}
& \bar{x} \frac{\partial u_{2}}{\partial \bar{x}}-u_{2}=(\Omega+\bar{x}) \sin \bar{z}-\varepsilon(8-\beta)(\Omega+\bar{x})\left(\Omega^{2}-2 \Omega \bar{x} \ln \bar{x}-\bar{x}^{2}\right)+ \\
& +2 \varepsilon \gamma\left[1+4 \varepsilon(\Omega+\bar{x})^{2}\right](\Omega-\Omega \ln \bar{x}-\bar{x})
\end{aligned}
$$

where

$$
\begin{aligned}
& \gamma=4-\cos \bar{z}+4 \sin 2 \bar{z}-3 \cos 3 \bar{z} \\
& \beta=\sin \bar{z}+8 \cos 2 \bar{z}+9 \sin 3 \bar{z}
\end{aligned}
$$

Substituting $u_{2}=\bar{x} \psi_{1}$, we have 


$$
\begin{aligned}
& \frac{d \Psi_{1}}{d x}=\left(\frac{\Omega}{\bar{x}^{2}}+\frac{1}{\bar{x}}\right) \sin \bar{z}-\varepsilon(8-\beta)\left(\frac{\Omega^{3}}{\bar{x}^{2}}-\frac{2 \Omega^{2}}{\bar{x}} \ln \bar{x}-\Omega+\frac{\Omega^{2}}{\bar{x}}-2 \Omega \ln \bar{x}-\bar{x}\right)+ \\
& +2 \varepsilon \gamma\left[\frac{\Omega}{\bar{x}^{2}}-\frac{\Omega}{\bar{x}^{2}} \ln \bar{x}-\frac{1}{\bar{x}}+4 \varepsilon\left(\frac{\Omega^{3}}{\bar{x}^{2}}+\frac{\Omega^{2}}{\bar{x}}-\Omega-\frac{\Omega^{3}}{\bar{x}^{2}} \ln \bar{x}-\frac{2 \Omega^{2}}{\bar{x}} \ln \bar{x}-\Omega \ln \bar{x}-\bar{x}\right)\right] .
\end{aligned}
$$

Then, for the function $u_{2}$ we have

$$
\begin{aligned}
& u_{2}(\bar{x}, \bar{z})=(\bar{x} \ln \bar{x}-\Omega) \sin \bar{z}+ \\
& +\varepsilon(8-\beta)\left(\Omega^{3}-\Omega \bar{x}^{2}+\frac{1}{2} \bar{x}^{3}+\Omega \bar{x} \ln ^{2} \bar{x}-\Omega^{2} \bar{x} \ln \bar{x}+2 \Omega \bar{x}^{2} \ln \bar{x}\right)+ \\
& +2 \varepsilon \gamma\left[\Omega \ln \bar{x}-\bar{x} \ln \bar{x}+4 \varepsilon\left(\Omega^{2} \bar{x} \ln \bar{x}+\Omega^{3} \ln \bar{x}-\Omega^{2} \bar{x} \ln ^{2} \bar{x}-\Omega \bar{x}^{2} \ln \bar{x}-\frac{1}{2} \bar{x}^{3}\right)\right]+ \\
& +C_{2}(\overline{\mathrm{z}}) \cdot \bar{x} .
\end{aligned}
$$

For boundedness of the function $u_{2}$, it is necessary and sufficient that

$$
C_{2}(\bar{z})=0
$$

Rewrite the last from the system of Eq.(3.14) with the results obtained

$$
\bar{x} \frac{\partial v_{2}}{\partial \bar{x}}=B_{3}-u_{1}
$$

whence

$$
B_{3}=0
$$

and hence, we obtain

$$
\mathrm{v}_{2}(\bar{x}, \bar{z})=\ln x \cdot(\cos \bar{z}-1)-\varepsilon \gamma\left[\Omega^{2} \ln \bar{x}-2 \Omega \bar{x}(\ln \bar{x}-1)-\frac{1}{2} \bar{x}^{2}\right] .
$$

By continuing this procedure, it is easy to get $\mathrm{A}_{4}$ etc. Limiting our calculations with the terms $O\left(\mu^{2}\right)$, we form Eqs (3.11) and (3.12) as

$$
\begin{aligned}
& x=\bar{x}+\mu(1-\cos \bar{z})+\mu \varepsilon \gamma\left(\Omega^{2}-2 \Omega \bar{x} \ln \bar{x}-\bar{x}^{2}\right)+\mu^{2}(\bar{x} \ln \bar{x}-\Omega) \sin \bar{z}+ \\
& +\mu^{2} \varepsilon(8-\beta)\left(\Omega^{3}-\Omega \bar{x}^{2}+\frac{1}{2} \bar{x}^{3}+\Omega \bar{x} \ln ^{2} \bar{x}-\Omega^{2} \bar{x} \ln \bar{x}+2 \Omega \bar{x}^{2} \ln \bar{x}\right)+ \\
& +2 \mu^{2} \varepsilon \gamma\left[\Omega \ln \bar{x}-\bar{x} \ln \bar{x}+4 \varepsilon\left(\Omega^{2} \bar{x} \ln \bar{x}+\Omega^{3} \ln \bar{x}-\Omega^{2} \bar{x} \ln ^{2} \bar{x}-\Omega \bar{x}^{2} \ln \bar{x}-\frac{1}{2} \bar{x}^{3}\right)\right]
\end{aligned}
$$




$$
\begin{aligned}
& z=\bar{z}+\mu^{2} \ln \bar{x}(\cos \bar{z}-1)-\mu^{2} \varepsilon \gamma\left[\Omega^{2} \ln \bar{x}-2 \Omega \bar{x}(\ln \bar{x}-1)-\frac{1}{2} \bar{x}^{2}\right] \\
& \bar{x}^{\prime}=-\mu \bar{x}-\mu^{2}\left[1+4 \varepsilon(\Omega+\bar{x})^{2}\right] \\
& \bar{z}^{\prime}=\mu^{2}(\Omega+\bar{x})
\end{aligned}
$$
we obtain

By integrating the third and fourth of Eqs (3.23) and taking into account the initial conditions (3.10),

$$
\begin{aligned}
& \bar{x}=D \cdot \frac{1+A e^{-\mu \alpha s}}{1-A e^{-\mu \alpha s}}-B, \\
& \bar{z}=\mu^{2}(\Omega-\sigma) \cdot s+k \ln \left(1-A e^{-\mu \alpha s}\right)-k \ln (1-\mathrm{A}),
\end{aligned}
$$

where

$$
\begin{aligned}
& \alpha=\sqrt{(1+8 \mu \varepsilon \Omega)^{2}-16 \mu^{2} \varepsilon\left(1+4 \mu \varepsilon \Omega^{2}\right)} ; \quad \mathrm{D}=\frac{\alpha}{8 \varepsilon \mu} ; \\
& B=\frac{1+8 \mu \varepsilon \Omega}{8 \mu \varepsilon} ; \quad \sigma=\frac{(1+8 \mu \varepsilon \Omega)-\alpha}{8 \mu \varepsilon} ; \quad k=\frac{1}{4 \varepsilon} ; \\
& A=\frac{1-\alpha}{1+\alpha} .
\end{aligned}
$$

Going back to the variables $t=\frac{\mu^{2}}{\omega_{0}} s$ and $\varphi=z$ and on the basis of formulas (3.23) and (3.24) we plot the graphs of the angular velocity on the rotation angle of the motor shaft (guiding link) with various parameters of the shaking table orthogonal mechanism. Calculations have been performed at the following parameters

$$
\begin{aligned}
& a=600 \mathrm{~N} \cdot \mathrm{m}, \quad b=300 \mathrm{~N} \cdot \mathrm{m} \cdot \mathrm{s}, \quad M=70 \mathrm{~kg}, \quad m=2 \mathrm{~kg}, \quad m_{l}=2.5 \mathrm{~kg}, \\
& m_{2}=3 \mathrm{~kg}, \quad m_{3}=3.5 \mathrm{~kg}, \quad l=0.1 \mathrm{~m}, \quad l_{1}=1 \mathrm{~m}, \quad l_{2}=2 \mathrm{~m} \text {. }
\end{aligned}
$$

In Fig. 2 line 1 is built on the results of the numerical solution of Eqs (3.8), and line 2 - on the result of analytical solutions. Similar curves give an indication of closeness of the analytical and numerical solutions' results. Graphs of the angular velocity of the motor shaft (guiding link) $\dot{\varphi}$ on the angle of rotation $\varphi$ for different values of the load mass $M$ are shown in Fig.3; for different values of the guiding link length - Fig.4; for different values of the engine torque - Fig.5. The graphs show that the shaft angular velocity values (guiding link) oscillate around its mean value. 
The amplitude of oscillograms depends on the values of the counterweight mass and guiding link length. The mean value of the angular velocity depends only on the value of the motor torque (Fig.5). Figure 6 shows the excitation region boundaries for rotational motion of the engine, built by formula (3.6).

Figure 7 shows the oscillograms of the horizontal (line 1) and vertical (line 2) oscillations of the orthogonal mechanism relative to the average values of the coordinates $x$ and $y$.

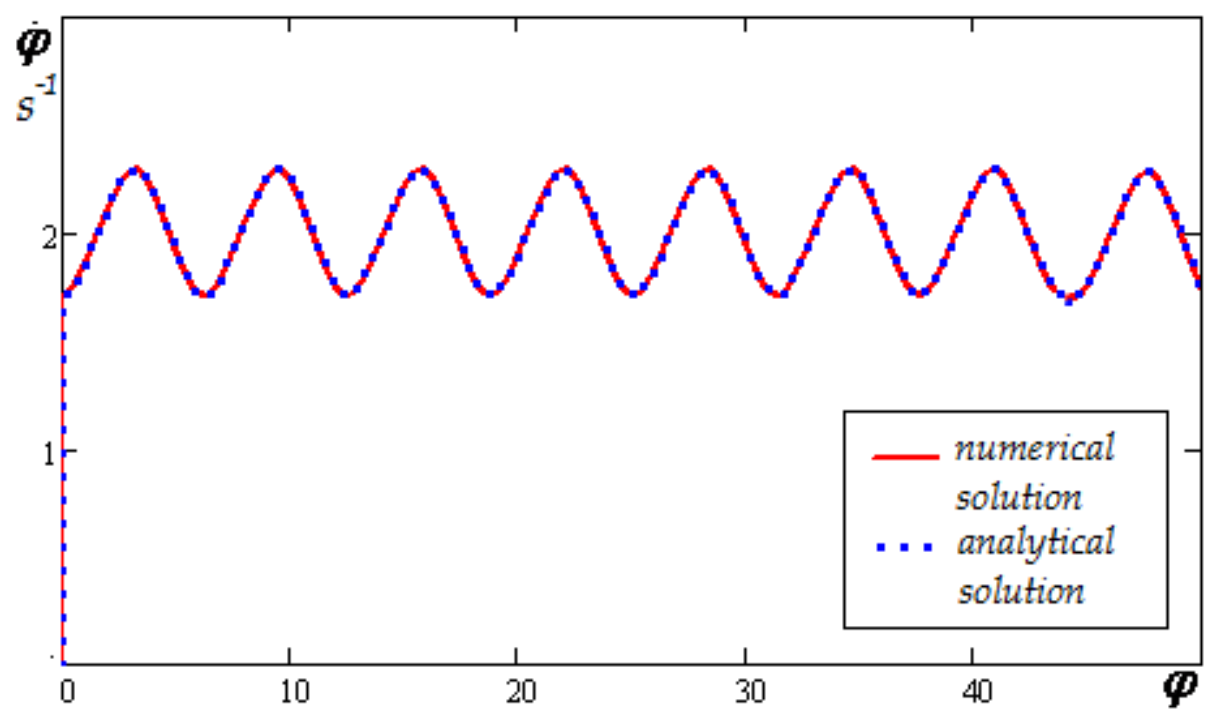

Fig.2. The dependence of the angular velocity on the shaft rotational angle (guiding link).

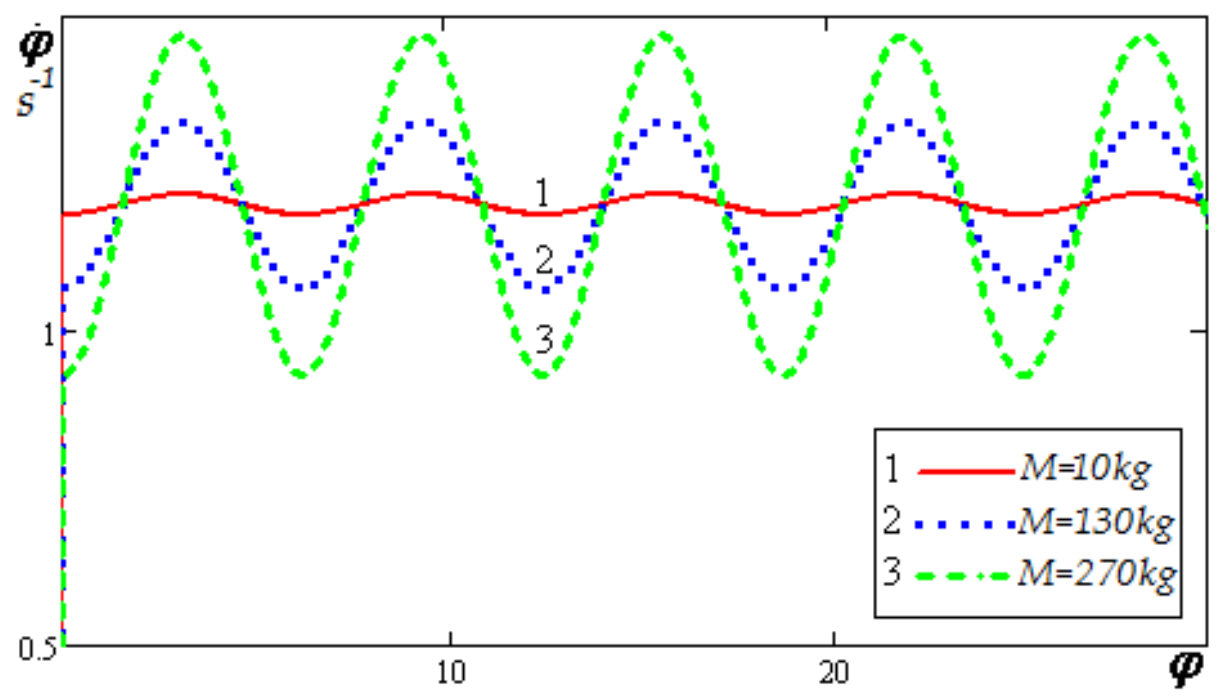

Fig.3. The dependence of the angular velocity on the shaft rotational angle (guiding link) at different values of the load mass. 


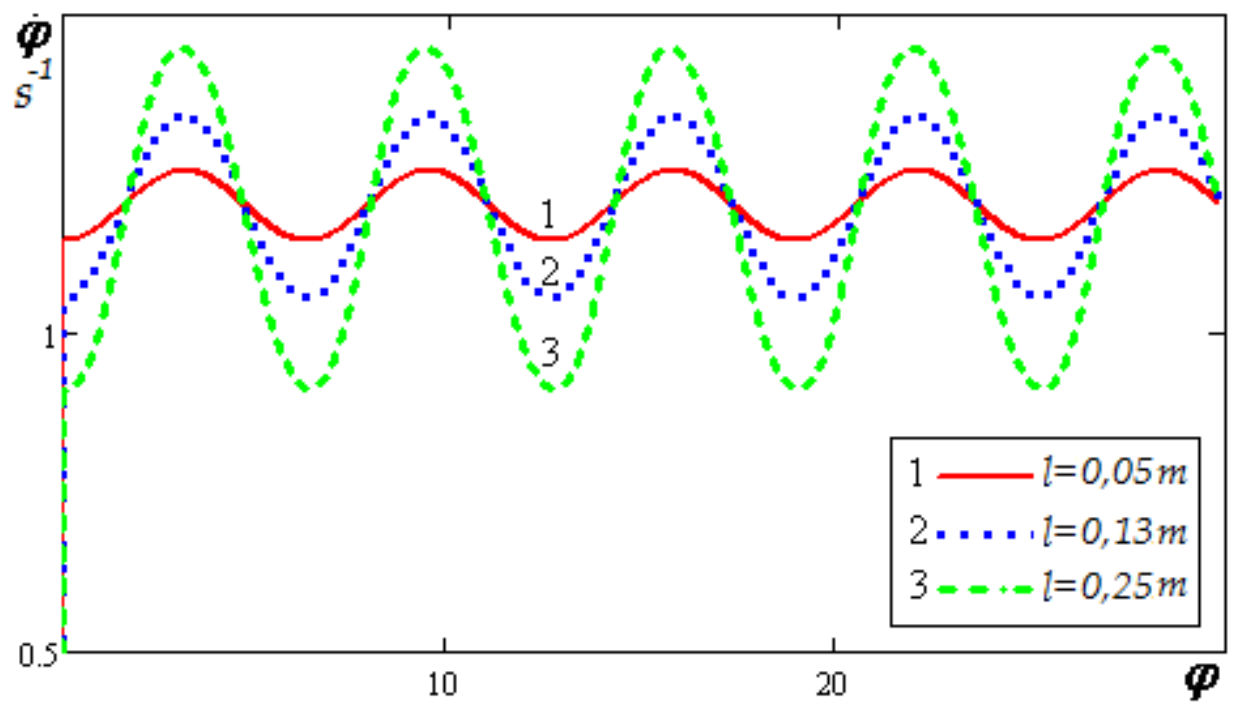

Fig.4. The dependence of the angular velocity on the shaft rotational angle (guiding link) at different values of the guiding link length.

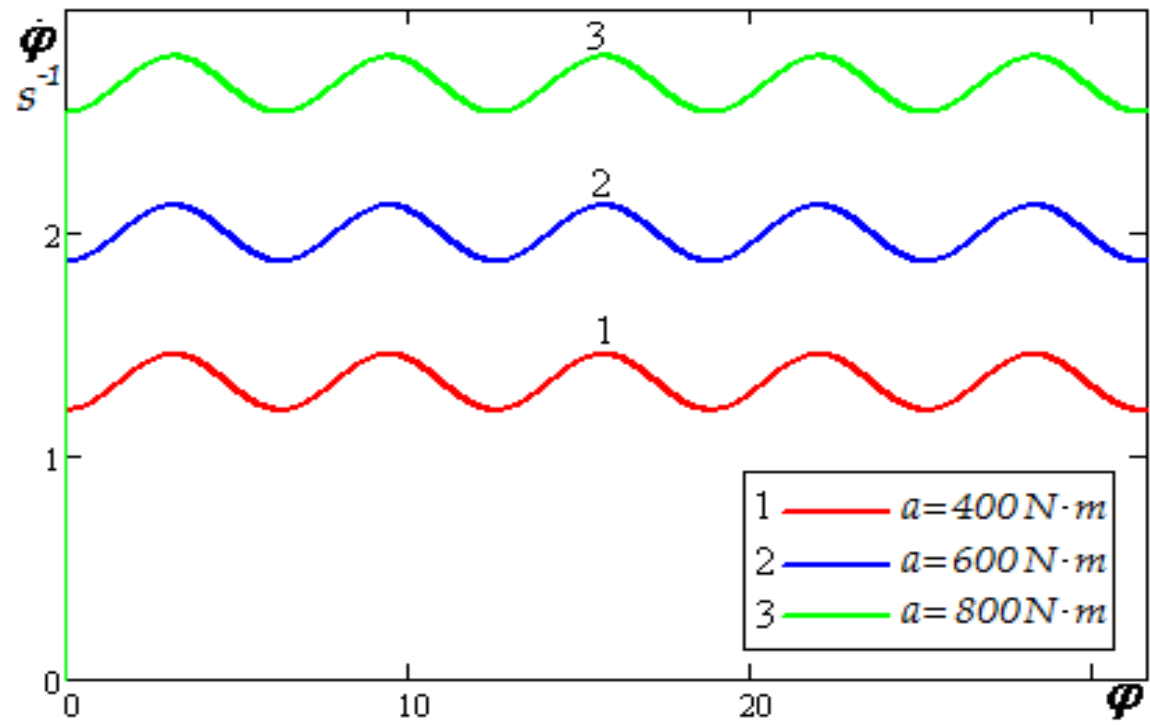

Fig.5. The dependence of the angular velocity on the shaft rotational angle (guiding link) at different values of the engine torque. 


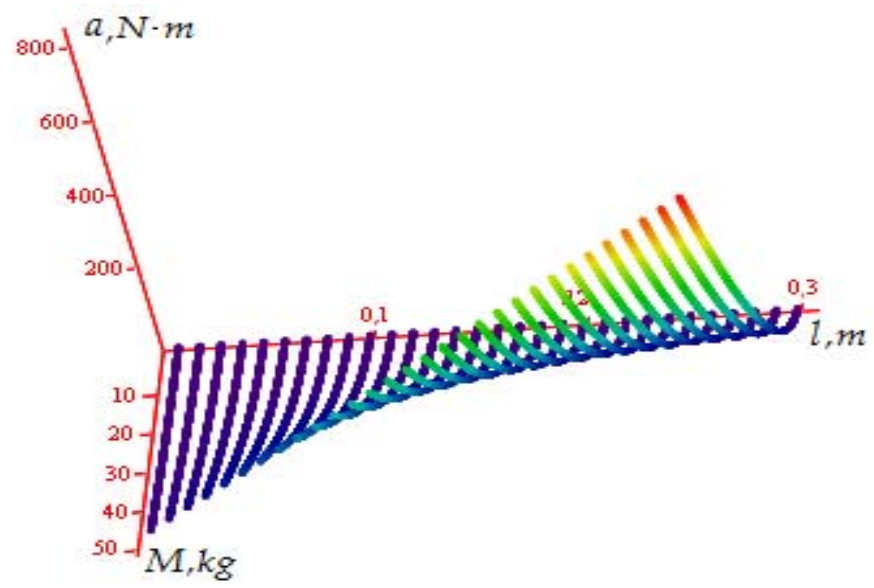

Fig.6. Excitation region boundaries for rotational motion of the motor shaft (guiding link).

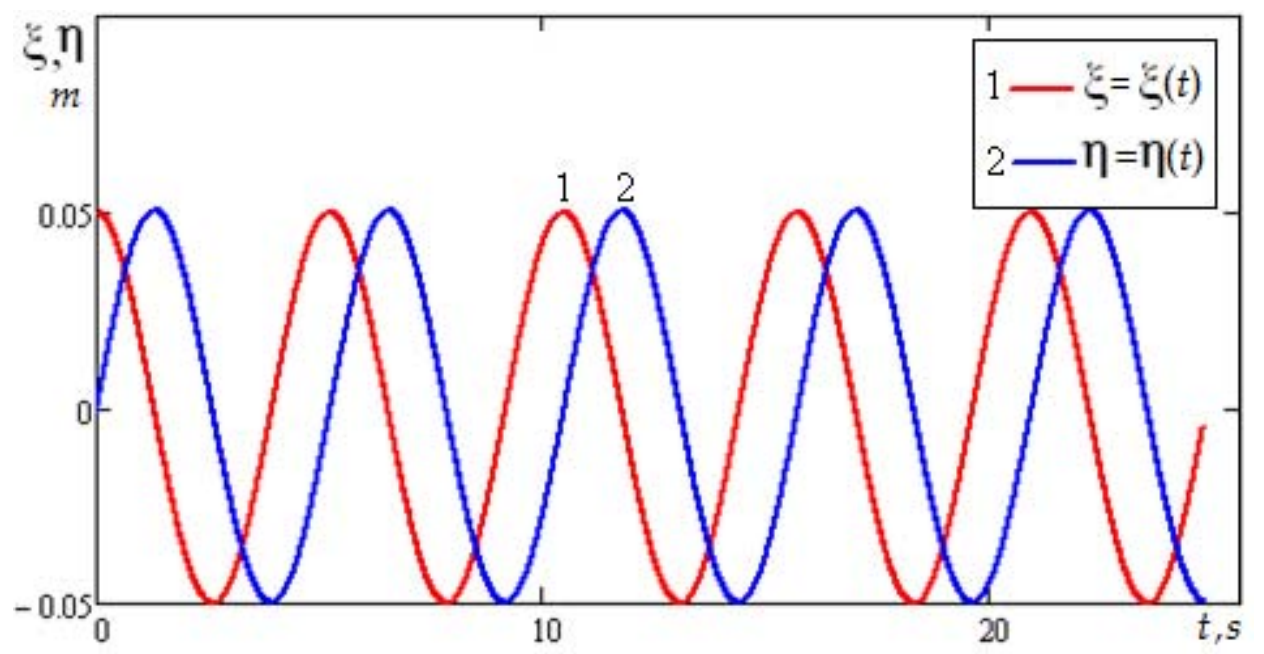

Fig.7. Graphs of horizontal and vertical oscillations of the orthogonal mechanism.

\subsection{Stability of motion}

Let us consider the stationary regimes of rotational motion of the orthogonal mechanism, which are given in Eq.(3.24). To do this, we form the variational equations for the system (3.23).

Set

$$
\begin{aligned}
& \bar{x}=\bar{x}^{*}+q, \\
& \bar{z}=\bar{z}^{*}+p,
\end{aligned}
$$

where $\bar{x}^{*}$ and $\bar{z}^{*}$ - stationary solutions of Eqs (3.23), that under the initial conditions: $s=0, \bar{z}=0, \bar{z}^{\prime}=0$ have the form

$$
\bar{x}^{*}=\mathrm{D} \frac{1+A e^{-\mu \alpha s}}{1-A e^{-\mu \alpha s}}-B,
$$




$$
\bar{z}^{*}=\mu^{2}(\Omega-\sigma) \cdot s+k \ln \left(1-A e^{-\mu \alpha s}\right)-k \ln (1-\mathrm{A})
$$

Substituting (3.25) to (3.23) linearizing with respect to variations $q$ and $p$, we get

$$
\begin{aligned}
& q^{\prime}=-\mu\left[1+8 \mu \varepsilon\left(\Omega+\bar{x}^{*}\right)\right] q, \\
& p^{\prime}=\mu^{2} q .
\end{aligned}
$$

Integrals of the equations system (3.26) will take the form

$$
\begin{aligned}
& q=\frac{C e^{-\mu \alpha s}}{\left(1-A e^{-\mu \alpha s}\right)^{2}}, \\
& p=-\frac{\mu C}{\alpha A} \frac{1}{\left(1-A e^{-\mu \alpha s}\right)} .
\end{aligned}
$$

Stability condition has the form of the inequality

$$
1+16 \mu \varepsilon(\Omega-\mu) \succ 0 .
$$

\section{Conclusion}

The dynamics of the orthogonal mechanism with a nonideal energy source in the presence of a shaking table fixed counterweight is studied. It is found that the angular velocity value of the slow-speed motor shaft (guiding link) oscillates around its average value according to the harmonic law. The amplitude of deviation of the angular velocity from its mean value depends on the counterweight mass and the guiding link length. In turn, the mean value of the angular velocity depends on the motor torque. A criterion for the stability of slow-motion guiding link of the shaking table orthogonal mechanism is established. Results, obtained in the theoretical studies, can be successfully used for the design of vibration vehicles with orthogonal mechanisms.

\section{Nomenclature}

$a$ and $b-$ constant coefficients depending on the parameters of the engine

$f$ - coefficient of sliding friction

$J \quad$ - link moment of inertia 1

$k-$ coefficient of friction, rotation

$l, l_{1}, l_{2}$ - lengths of links $1,2,3$, respectively

$M$ - counterweight mass

$M_{D}-$ moment of the driving forces (the torque on the shaft of a motor)

$m, m_{1}, m_{2}$ and $m_{3}$ - mass of the links $1,2,3$ and 4

$X, Y$ - coordinates of the articulated link $\mathrm{C}$

$\xi, \eta$ - deviations coordinates of the articulated link $\mathrm{C}$ from their average value

$\varphi \quad$ - shaft rotational angle (guiding link) 
$\dot{\varphi}-$ angular velocity of the shaft rotational (guiding link)

$\tau$ - dimensionless time

\section{References}

Alifov A.A. and Frolov K.V. (1985): Interaction of Non-Linear Oscillation Systems with Sources of Energy. - Moscow: Nauka.

Arakelian V. and Daham M. (2001): Partial shaking moment balancing of fully force-balanced linkage. - Mechanism and Machine Theory, vol.8, No.36, pp.1241-1252.

Azbel G.G., Blekhman N.I., Bykhovskiy I.I. and others (1981): Vibration processes and machines. - Volume 4, edition of E.E. Levendel, Vibrations in Technique (guide), Moscow, Mechanic Engineering.

Baksys B. and Liutkauskiene K. (2008): Displacement of the body on the oscillatory plane. - Mechanika, vol.3, No.71, pp.1-37.

Baksys B. and Liutkauskiene K. (2010): Manipulation of apart on a vibrating plane under controlled dry friction. Mechanika, vol.3, No.83, pp.28-33.

Bisembayev K. and Iskakov Zh. (2012): Mathematic model of the orthogonal mechanism of the press machine shaking table. - Bulletin of The Kazakh National Teachers Training University named after Abai, vol.3, No.39, pp.32-38.

Bogolyubov N.N. and Mitropolskiy Yu.A. (1974): Asymptotic Methods of the Non-Linear Oscillations Theory. Moscow: Science.

Dorinin V.I. and Danshin Yu.V. (1993): Dynamic synthesis of flat balanced leverage mechanisms. - Khabarovsk, DVG APS.

Drakunov Yu.M. and Tuleshov E.A. (2007): Dynamics of a shaking table in generalized functions. - Proceedings of the International Scientific Technical Conference Yerzhanov's Readings II, pp.132-134.

Feng B., Morita N. and Torii T. (2004): Dynamic Optimum Design for Path Generation of Planar Linkage. Proceedings of $11^{\text {th }}$ World Congress in Mechanism and Machine Science, Tvanjin.

Gulyayev V.I., Bazhenov V.A. and Popov S.L. (1989): Applied Tasks of the Theory of Non-Linear Oscillations of Mechanical Systems. - Moscow: Higher School.

Hong-Sen Yan and Ren-Chung Soong (2001): Kinematic and dynamic design of four-bar linkages by links counterweighing with variable input speed. - Mechanism and Machine Theory, vol.9, No.37, pp.1051-1071.

Kononenko V.O. (1964): Oscillation Systems with Restricted Excitation. - Moscow: Nauka.

Krivtsov A.M. (2000): Influence of the torque of restricted power on the stability of stationary motions of nonsymmetrical spin. - Mechanics of Solid Body, vol.2, pp.33-43.

Moyseyev N.N. (1969): Asymptotic Methods of Non-Linear Mechanics. - Moscow: Science.

Tuleshov A.K. and Tuleshov E.A. (2012): Solution of shaking table dynamics equations in the class of generalized functions using the iteration method. - Proceedings of the International Scientific Conference Modern Achievements in the Science and Education.

Received: May 12, 2014

Revised: May 22, 2014 